\title{
Continuous versus pulsed microwave ablation in the liver: any difference in intraoperative pain scores?
}

\author{
Dimitrios Filippiadis ${ }^{a}$, Argyro Maziotia ${ }^{a}$ George Velonakis ${ }^{a}$, Athanasios Tsochantzis ${ }^{a}$, Nevio Tosoratti ${ }^{b}$, \\ Alexis Kelekis ${ }^{a}$, Nikolaos Kelekis ${ }^{a}$ \\ "Attikon" University Hospital, Medical School, National and Kapodistrian University of Athens; HS Hospital Service \\ SpA, Aprilia (LT), Italy
}

\section{Abstract}

a $2^{\text {nd }}$ Department of Radiology, "Attikon" University General Hospital, Medical School, National and Kapodistrian University of Athens, Greece (Dimitrios Filippiadis, Argyro Mazioti, George Velonakis, Athanasios Tsochantzis, Alexis Kelekis, Nikolaos Kelekis); ${ }^{\mathrm{b}} \mathrm{R} \& \mathrm{D}$ Manager, HS Hospital Service SpA, Aprilia (LT), Italy (Nevio Tosoratti)

Conflict of Interest: Nevio Tosoratti is an employee of H.S. Hospital Service SpA, the company manufacturing the MWA apparatus used in the study. The authors alone are responsible for the content and writing of the paper. The rest authors declare that they have no conflict of interest

Correspondence to: Dimitrios K. Filippiadis, Assistant Professor of Diagnostic and Interventional Radiology, $2^{\text {nd }}$ Department of Radiology, "Attikon" University General Hospital, Medical School, National and Kapodistrian University of Athens, 1 Rimini St., 12462 HAIDARI, Athens, Greece, e-mail: dfilippiadis@yahoo.gr

Received 30 June 2020; accepted 24 August 2020; published online 20 November 2020

DOI: https://doi.org/10.20524/aog.2020.0557

\section{Introduction}

Microwave (MW) ablation is an increasingly applied technique for the treatment of malignant tumors, achieving high local tumor control rates in both primary and secondary lesions, irrespectively of the target's histology; the resultant larger ablation volumes may be achieved in shorter treatment times compared to any other thermal ablation modality [1-8]. The typical MW ablation zone obtained through a single energy delivery of a single probe, dwelling in a given position for the entire treatment duration, is an elongated ellipsoid with rotational symmetry around the probe's axis, whose aspect ratio (defined as: $\mathrm{S}=\mathrm{D} / \mathrm{L}$, where $\mathrm{L}$ is the maximum ablation size along the probe axis and $\mathrm{D}$ is the maximum ablation size perpendicular to it) generally ranges from $0.55-0.75$ [9]. Over 
the last decade, in an attempt to enhance ablation sphericity (i.e., taking S closer to 1), several MW vendors have deployed a variety of technical solutions, from new antenna designs to multi-probe configurations or modification of the standard MW delivery scheme (i.e., continuous provision of a fixed power $\mathrm{P}$ for the entire treatment duration $\mathrm{T}$ ) [10-12].

Anesthesiology and analgesia protocols in the routine practice of percutaneous ablation are widely variable across centers and clinicians, ranging from intravenous analgesia to conscious or deep sedation and even up to general anesthesia. Significant factors include, but are not limited to, availability of the anesthesiologist, personal preferences of both the operator and the anesthesiologist, as well as patient's comorbidities and performance status.

The purpose of this study was to prospectively evaluate pain tolerability during liver MW ablation with continuous or pulsed energy delivery modes, by comparing the patients' self-reported pain scores immediately upon completion of the ablation session.

\section{Patients and methods}

All patients were informed about the interventional technique to be used, with its possible benefits and complications, and signed a written informed consent form prior to the procedure and the study. The study protocol conformed to the ethical guidelines of the 1975 Declaration of Helsinki and received approval by the institution's human research committee prior to patients' enrollment and treatment.

\section{Patient selection and evaluation}

Prior to percutaneous MW ablation, a cohort of 20 patients was randomized on a 1:1 basis between continuous (CM group) and pulsed (PM group) treatment. The study duration was 12 months. Lesions were diagnosed based on either contrast-enhanced magnetic resonance imaging (MRI) or positron emission tomography findings. Patients were selected for MW ablation by a multidisciplinary team of medical, radiation, surgical and interventional oncologists. Each patient underwent laboratory tests (including renal function and coagulation tests) at least $24 \mathrm{~h}$ prior to the percutaneous ablation session. Patient and tumor characteristics, treatment algorithm and parameters, intra- and postoperative complications, and intraoperative pain scores were recorded for each procedure. All ablation sessions were performed by 2 interventional radiologists (one with 20 and one with 10 years of experience); neither was blind to the energy delivery mode applied. Pain score questions were asked by a nurse within the first $30 \mathrm{~min}$ post ablation; the nurse was blind to the energy delivery mode applied.

The primary objective of the study was to compare the intraoperative pain between continuous and pulsed energy delivery modes; secondary objectives included local tumor control on follow-up imaging checks with MRI (including diffusion-weighted sequences and sequences after i.v. gadolinium injection).

Inclusion criteria included patients $\geq 18$ years old with primary or metastatic hepatic disease, confirmed either by prior biopsy or through imaging (defined as new or growing nodules in cases of histological proven primary cancer); up to 3 hepatic lesions with a maximum size of $3 \mathrm{~cm}$; Karnofsky Performance Scale score $\geq 60$, coagulation parameters within normal limits and a life expectancy of $>3$ months. All included patients and lesions had to be evaluable over the 6-month follow up. All patients were referred to the study by the multidisciplinary tumor board. Exclusion criteria included a baseline pain level $\geq 2$ prior to the ablation procedure, uncontrollable primary or metastatic disease outside the liver, patient's non-compliance, uncontrollable international normalized ratio, systematic or local infection, poorly controlled ascites, expected survival less than 3 months, Eastern Cooperative Oncology Group score less than 3, and the presence of a medical or psychiatric illness that would preclude informed consent or follow up.

For the randomization process, we used the following procedure: when a patient met the inclusion criteria, he or she was assigned a number (the first patient referred to our department was assigned number 1 , the second patient, number 2 , the third patient, number 3 , etc.). Odd-numbered patients were assigned to the CM group and even-numbered patients to the PM group.

\section{Percutaneous ablation procedure}

MW ablation was always performed in an inpatient setting. Computed tomographic (CT) guidance with sequential scanning ( $120 \mathrm{Kv}$ peak, $240 \mathrm{mAs}$ wavelength and $2 \mathrm{~mm}$ slice thickness) was used for planning, targeting and intraprocedural modification during the ablation session. The analgesia protocol was the same throughout the cohort: 30 min prior to ablation tramadol was injected intravenously, diluted in $100 \mathrm{~mL}$ normal saline, whilst acetaminophen was administered during the ablation session to treat intraprocedural pain (amounts of both analgesics were based on each patient's weight) [13]. Under local sterility, MW ablation was performed via a percutaneous approach in all cases. After the initial CT scan, a skin entry point was selected. All treatments were performed using the same MW ablation equipment (HS AMICA, HS Hospital Service SpA, Rome, Italy), comprising a $2450 \mathrm{MHz}$ solid-state generator with integrated peristaltic pump (AMICA-GEN) and internally cooled MW applicators (AMICA-PROBE). The MW probe was inserted in the lesion of interest through sequential CT scans (Fig. 1). In all cases included in the present study ablation was performed using a single MW antenna. Once in the correct location, the ablation session was set up and performed according to a specific treatment plan, mainly stemming from a review of the coagulation charts provided by the manufacturer in consideration of the tumor size and location and the desired safety margin. These charts, based on 

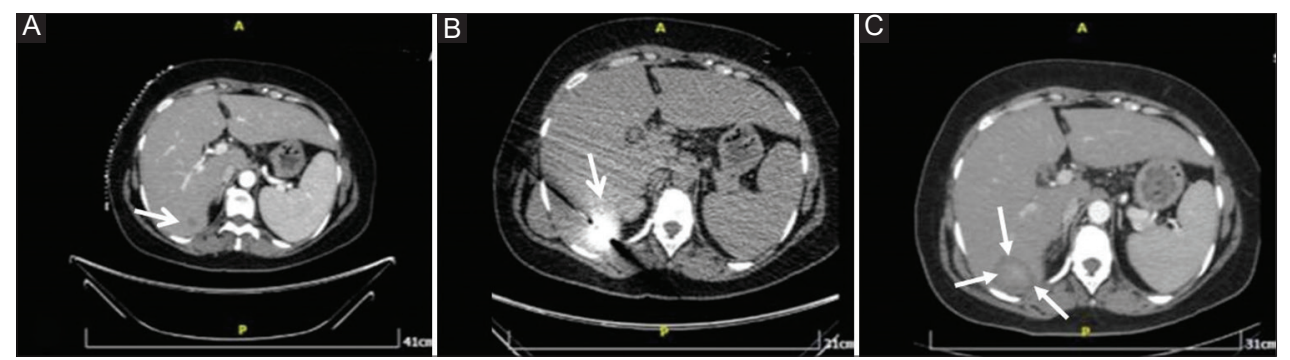

Figure 1 A 45-year-old woman with breast cancer and a single metastatic lesion in the liver, treated by percutaneous microwave ablation (pulsed mode). (A) Computed tomography (CT) image in the axial plane shows the lesion (arrow) in segment VI of the liver. (B) CT image in the axial plane shows the microwave antenna (arrow) in the final position. (C) CT image in the axial plane shows the ablation zone (arrows) immediately post ablation

lab experiments on ex vivo bovine liver, provide for the ablation dimensions $(\mathrm{L} \times \mathrm{D})$ to be expected-with all the inherent limitations of an ex vivo animal model-for given settings of the energy delivery scheme (either continuous or pulsed, with $\mathrm{t}_{\mathrm{ON}}=4 \mathrm{sec}$ and $\mathrm{t}_{\mathrm{OFF}}=6 \mathrm{sec}$ ), the output power (up to $100 \mathrm{~W}$ in continuous mode and up to $140 \mathrm{~W}$ in pulsed mode) and the overall treatment time (up to $15 \mathrm{~min}$ ). Depending on the size of the lesion to be treated, the ablation protocols in the CM group provided for 40 or $60 \mathrm{~W}$ for either 5 or $10 \mathrm{~min}$, while in the PM group all sessions lasted $10 \mathrm{~min}$ at either 100 or $120 \mathrm{~W}$. In either trial arm, whenever deemed necessary, the MW antenna was repositioned and a second ablation session was performed, so as to ensure that the final ablation completely encompassed both the target tumor and an annular safety zone around it at least $5 \mathrm{~mm}$ thick.

\section{Statistical analysis}

Study outcomes were numerically presented as mean \pm standard deviation. Pain scores in the 2 trial arms were compared using the paired-samples Student's $t$-test. P-values $<0.05$ were considered to indicate a statistically significant difference. Multivariate analysis was performed in order to evaluate the role of confounding factors. Sample size calculation using a pain difference of 3 numeric visual scale (NVS) Units (considered clinically significant) was also performed, suggesting a sample of 14 patients for a power of $80 \%$. Statistical analyses were performed using SPSS Statistics 22 (IBM Corp. Released 2013. IBM SPSS Statistics for Windows, Version 22.0. Armonk, NY: IBM Corp).

\section{Outcome measures}

CT (contrast enhanced, in the arterial and portal venous phase of enhancement) was used to assess both the ablation zone size and the potential immediate complications at the end of the ablation treatment (Fig. 2). Exactly $30 \mathrm{~min}$ after the completion of ablation sessions, all patients completed a questionnaire in which they assigned a numeric pain intensity score from 0 (no pain) to 10. Patients were kept under observation for $24 \mathrm{~h}$ and eventually discharged. We evaluated technical success, complication rates and intraoperative pain scores. Pain score was evaluated using a brief pain inventory containing questions about pain intensity; answers were provided in terms of NVS [14]. Technical success was defined as complete coverage of the lesion by the ablation zone immediately after the procedure, plus a safety margin of at least $5 \mathrm{~mm}$ (as depicted by CT scan before and after iodinated contrast medium injection in the arterial and portal venous phases of enhancement). Complications were defined according to the Cardiovascular and Interventional Radiological Society of Europe (CIRSE) classification system [15].

\section{Results}

The male:female ratio in both groups was 8:2. The mean age was $69.60 \pm 9.55$ years in the CM group and $63.30 \pm 11.40$ years in the $\mathrm{PM}$ group $(\mathrm{P}=0.197)$. The $\mathrm{PM}$ group included 10 patients with 11 lesions: 5 hepatocellular carcinomas (HCCs) and 6 metastases (colon $n=5$ and breast $n=1$ ); 4/11 (36.6\%) tumors were subcapsular. The CM group included 10 patients with 10 hepatic lesions: 5 HCCs and 5 metastases (colon $\mathrm{n}=2$, pancreas $\mathrm{n}=1$, breast $\mathrm{n}=1$, bronchogenic $\mathrm{n}=1) ; 3 / 10(30 \%)$ tumors were sub-capsular (i.e., $\leq 2 \mathrm{~cm}$ from the liver capsule). Five of 10 patients in the CM group and 6/10 in the PM group received systematic therapy during the ablation and study period. All patients remained hospitalized for the night following ablation and exited the hospital next morning.

The mean procedure time, including local anesthesia, placement of MW antenna, ablation and post-procedural CT evaluation was $53.5 \pm 20.90 \mathrm{~min}$ in the PM group vs. $58.5 \pm 17.44 \mathrm{~min}$ in the CM group $(\mathrm{P}=0.279)$ (Fig. 2). The mean size of the lesions was $2.81 \pm 0.95 \mathrm{~cm}$ in the $\mathrm{PM}$ group and $2.81 \pm 0.85 \mathrm{~cm}$ in the CM group $(\mathrm{P}=0.984)$.

On a per-lesion basis, technical success was achieved in all evaluable tumors in both groups. There was no repeat treatment of an index tumor. Local recurrence-free response (local tumor efficacy) of the treated lesions at 6 months was $90.90 \%(10 / 11)$ in the PM group and 100\% in the CM group. The recurrent tumor in the PM group was depicted in a lesion next to a vessel and was retreated with continuous-mode MW ablation. On a per patient basis, at 6-month follow up in the 


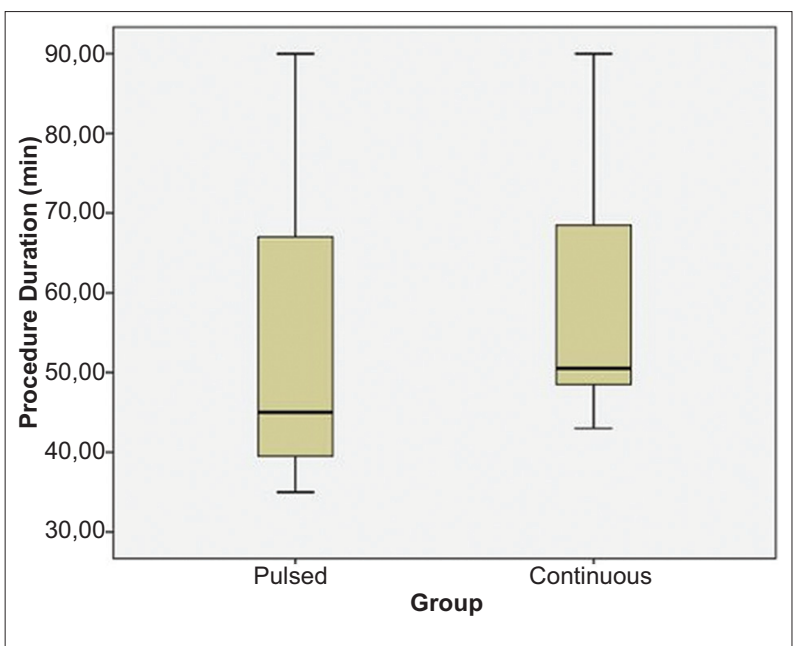

Figure 2 Chart illustrating mean duration values of the ablation session between the 2 groups. There was no statistically significant difference $(\mathrm{P}=0.279)$

PM Group overall treatment response was complete for $90 \%$ (9/10) in the PM group and 100\% (10/10) in the CM group.

The mean pain score was $8.17 \pm 1.850$ in the CM group and $4.50 \pm 1.567$ pain units in the PM group (Fig. 3 ). There was a statistically significant difference of $3.667 \pm 2.807$ pain units $(\mathrm{P}=0.001)$. No complications were noted in the CM group, whereas in the PM group there were 2 grade I complications according to the CIRSE classification system (small, perihepatic hemorrhagic fluid collections). During the follow-up period there were no deaths related to the procedure or to disease progression.

\section{Discussion}

A newer approach to sphericity enhancement involves the modification of the standard MW delivery scheme (i.e., continuous provision of a fixed power $\mathrm{P}$ for the entire treatment duration T). A few authors have proposed MW energy pulsing, i.e., intermittent energy administration characterized by a succession of active delivery periods, each of duration $t_{\mathrm{ON}}$, and rest periods, each of duration $\mathrm{t}_{\mathrm{OFF}}$ (Fig. 1A) [12]. After a given treatment time $\mathrm{T}$, the amount of energy deposited in the patient during a pulsed ablation procedure is: $\mathrm{E}_{\mathrm{d}}=\mathrm{P}^{\star} \mathrm{T}^{\star} \mathrm{DC}$, where $\mathrm{P}$ is the power intensity delivered during active periods and $\mathrm{DC}=\mathrm{t}_{\mathrm{ON}} /\left(\mathrm{t}_{\mathrm{ON}}+\mathrm{t}_{\mathrm{OFF}}\right)$ is the percentage active time (i.e., duty cycle); therefore, as $\mathrm{DC}<1$, higher $\mathrm{P}$ values are needed to compensate for rest periods and to warrant the same final energy deposition, compared to a continuous delivery scheme of equal duration. The ability of pulsing algorithms to generate more spherical ablations relies on the possibility of differentiating the temperature profiles parallel and perpendicular to the MW probe axis: for internally cooled probes, during rest periods tissues lying closer to the probe shaft are cooled faster than tissues at the ablation periphery farther from the probe; for appropriate choices of $\mathrm{t}_{\mathrm{ON}}$ and $\mathrm{t}_{\mathrm{OFF}}$ (and provided shaft cooling

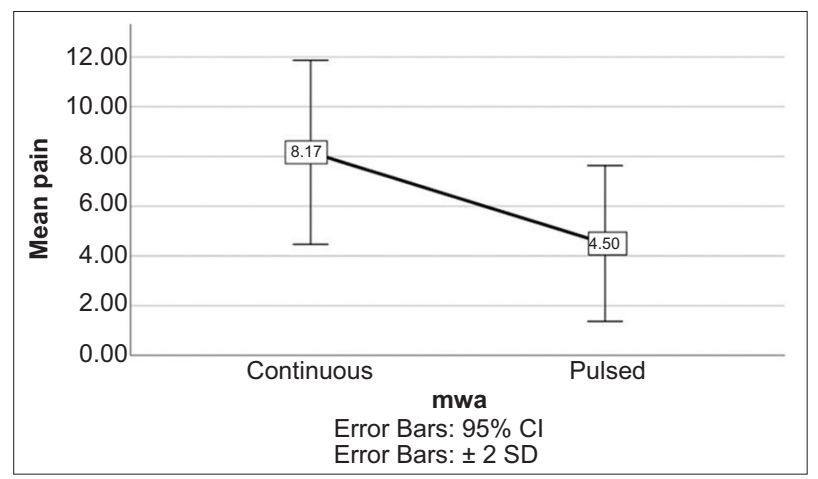

Figure 3 Chart illustrating mean pain score values between the 2 groups. There was a statistically significant difference of $3.667 \pm 2.807$ pain units $(\mathrm{P}=0.001)$

is adequately intensive and effective), this can be exploited to reduce the ablation length $\mathrm{L}$ while minimally affecting its transversal diameter $\mathrm{D}$, so as to yield an improved $\mathrm{S}=\mathrm{D} / \mathrm{L}$ aspect ratio (Fig. 1B).

Pulsing algorithms are a valid means for creating more uniform temperature gradients and less anisotropic thermal profiles within tissues. Since postprocedural pain at the ablation site is certainly correlated with inflammatory reactions, which in turn are triggered by the treatment-induced thermal profile, the less steep and more uniform temperature gradient associated with pulsing rather than continuous MW delivery schemes may indeed result in less painful procedures [16,17]. A paper by Haugh et al nicely highlights the different temperature profiles obtained using the $2 \mathrm{MW}$ delivery techniques under investigation: pulsed ablations resulted in much more uniform temperature maps around the emitting probe compared to continuous ablations [18]. In other words, the continuous mode allowed for higher temperatures closer to the emitting antenna but ended up with very similar temperatures compared to the pulsed mode at a given distance from the emitting tip: as the overall ablation zone is the ellipsoidal tissue volume contained within the $55^{\circ} \mathrm{C}$ isotherm induced by MW energy delivery during the treatment, both modalities proved equally capable of ablating similar tissue volumes, but pulsed ablation achieved this through a much more uniform temperature profile. One may infer (though not conclusively) that pulsed ablations provide for a "milder" treatment compared to continuous ablations, while depositing an equal amount of energy into tissues: this may account for a local reduction in temperaturetriggered biological mechanisms (such as inflammation, nervous stimulation, production of cytokines, etc.), which also correlate with pain response. Another potential explanation might associate pain with the ablation shape: since the ablation zone is much longer than it is wide using continuous ablation mode, one would expect that the ablation zone more often extended into the capsule and/or perivascular areas (which are the sensitive areas in the liver) using continuous vs. pulsed ablation mode.

There are several limitations to our study that should be taken into account. Pain scores are inherently subjective and may be biased by patients' concurrent disabilities. 
Additionally, the number of patients enrolled for the study was certainly small considering the wide histological variety of the treated tumors, potentially introducing further biasing factors. Furthermore, there are no long-term follow-up results concerning the efficacy and survival rates associated with the 2 different energy delivery modes. Finally, this paper considers only a small subset of the many available treatment protocols (in terms of power and time settings), for both the continuous and the pulsed energy delivery modes.

Our results provide preliminary evidence of significant intraoperative pain reduction in CT-guided MW liver ablation procedures compared to conventional continuous delivery schemes, with no difference in the outcome (i.e., complete tumor necrosis) for liver lesions $<3 \mathrm{~cm}$ in diameter. Hence, pulsed MW ablations appear to be an attractive option for patients with small to medium sized lesions undergoing liver ablation under i.v. analgesia, or whenever anesthesiologists are not available for the procedure. Future larger scale prospective trials with more circumscribed inclusion criteria are warranted in order to accumulate more robust and disease-specific data.

\section{Summary Box}

\section{What is already known:}

- Continuous-mode microwave (MW) ablation is an increasingly applied technique for the treatment of malignant tumors

- In an attempt to enhance ablation zone sphericity, a pulsed mode of MW ablation has been developed

\section{What the new findings are:}

- A pulsed mode of MW ablation can be an attractive alternative for percutaneous ablation of liver lesions $<3 \mathrm{~cm}$ under iv analgesia or mild sedation

- The pulsed mode of MW ablation seems to induce less intraoperative pain, while having similar safety rates compared to continuous mode for liver lesions $<3 \mathrm{~cm}$

- Both algorithms for MW energy delivery have comparable treatment effects in terms of 6-month local tumor control for liver lesions $<3 \mathrm{~cm}$

\section{References}

1. Filippiadis DK, Gkizas C, Chrysofos M, et al. Percutaneous microwave ablation of renal cell carcinoma using a high power microwave system: focus upon safety and efficacy. Int $J$ Hyperthermia 2018;34:1077-1081.
2. Shady W, Petre EN, Do KG et al. Percutaneous microwave versus radiofrequency ablation of colorectal liver metastases: ablation with clear margins $(\mathrm{AO})$ provides the best local tumor control. $J$ Vasc Interv Radiol 2018;29:268-275.

3. Potretzke TA, Ziemlewicz TJ, Hinshaw JL, et al. Microwave versus radiofrequency ablation treatment for hepatocellular carcinoma: a comparison of efficacy at a single center. J Vasc Interv Radiol 2016;27:631-638.

4. Macchi M, Belfiore MP, Floridi C, et al. Radiofrequency versus microwave ablation for treatment of the lung tumours: LUMIRA (lung microwave radiofrequency) randomized trial. Med Oncol 2017;34:96.

5. Deib G, Deldar B, Hui F, Barr JS, Khan MA. Percutaneous microwave ablation and cementoplasty: utility in the treatment of painful extra-osseous metastatic disease and myeloma. AJR Am J Roentgenol 2019;212:1377-1384.

6. Dodd GD $3^{\text {rd }}$, Dodd NA, Lanctot AC, Glueck DA. Effect of variation of portal venous blood flow on radiofrequency and microwave ablations in a blood-perfused bovine liver model. Radiology 2013;267:129-136.

7. Hinshaw JL, Lubner MG, Ziemlewicz TJ, Lee FT Jr, Brace CL. Percutaneous tumor ablation tools: microwave, radiofrequency, or cryoablation-what should you use and why? Radiographics 2014;34:1344-1362.

8. Bailey CW, Sydnor MK Jr. Current state of tumor ablation therapies. Dig Dis Sci 2019;64:951-958.

9. Hoffmann R, Rempp H, Erhard L, et al. Comparison of four microwave ablation devices: an experimental study in ex vivo bovine liver. Radiology 2013;268:89-97.

10. Alonzo M, Bos A, Bennett S, Ferral H. The Emprint ${ }^{\mathrm{m} w}$ ablation system with Thermosphere ${ }^{m}$ technology: one of the newer nextgeneration microwave ablation technologies. Semin Intervent Radiol 2015;32:335-338.

11. Harari CM, Magagna M, Bedoya M, et al. Microwave ablation: comparison of simultaneous and sequential activation of multiple antennas in liver model systems. Radiology 2016;278:95-103.

12. Bedoya M, del Rio AM, Chiang J, Brace CL. Microwave ablation energy delivery: influence of power pulsing on ablation results in an ex vivo and in vivo liver model. Med Phys 2014;41:123301.

13. Cornelis FH, Monard E, Moulin MA, et al. Sedation and analgesia in interventional radiology: Where do we stand, where are we heading and why does it matter? Diagn Interv Imaging 2019;100:753-762.

14. Mystakidou K, Mendoza T, Tsilika E, et al. Greek brief pain inventory: validation and utility in cancer pain. Oncology 2001;60:35-42.

15. Filippiadis DK, Binkert C, Pellerin O, Hoffmann RT, Krajina A, Pereira PL. CIRSE quality assurance document and standards for classification of complications: the CIRSE classification system. Cardiovasc Intervent Radiol 2017;40:1141-1146.

16. Amabile C, Ahmed M, Solbiati L, et al. Microwave ablation of primary and secondary liver tumours: ex vivo, in vivo, and clinical characterisation. Int J Hyperthermia 2017;33:34-42.

17. Andreano A, Galimberti S, Franza E, et al. Percutaneous microwave ablation of hepatic tumors: prospective evaluation of postablation syndrome and postprocedural pain. J Vasc Interv Radiol 2014;25:97-105.

18. Haugh C, Davidson ES, Estes NAM, Wang PJ. Pulsing microwave energy: a method to create more uniform myocardial temperature gradients. J Interv Card Electrophysiol 1997;1:57-65. 